\title{
Microgravity Biologics Crystallization Processes
}

Paul Reichert / Merck Research Laboratories / paul.reichert@merck.com

The International Space Station National Laboratory (ISS NL) offers a unique opportunity to perform bio-medical research and investigate pharmaceutical processes. Biologics are monoclonal antibodies (mAb), vaccines and biosimilars and have been approved for both life threatening and common ailments. Merck Research Laboratories in collaboration with the Center for the Advancement of Science in Space (CASIS) is investigating the growth of high quality $\mathrm{mAb}$ crystalline suspensions. In the area of biologic therapeutics, crystalline mAbs have applications in drug discovery, delivery, product development and manufacturing. By taking advantage of microgravity effects such as reduced sedimentation, minimal convection currents and reduced molecular diffusion rates, we hope to identify the key variables to produce high quality $\mathrm{mAb}$ crystalline preparations for earth processes. Merck Research Laboratories has several years of experience in microgravity crystallization experiments and has flown experiments on 11 space shuttle missions. These experiments resulted in the high yield production of crystals larger, more uniform and with a higher purity than the earth-grown samples. We will disclose the design and process of our current Sortie missions to the ISS aboard SpaceX-3, 6 and 10. Our SpaceX-CRS-10 experiment with Keytruda (pembrolizumab) focused on potential structure, purification and drug delivery applications. These applications can benefit patients through the development of biologic therapeutics which improve the safety, adherence, compliance and quality of life for patient \& caregivers. The results of these experiments as well as our plans for future missions will be disclosed. 